\title{
5 Mobilität
}

Dieses Kapitel wurde bei Erstveröffentlichung ohne die korrekte Creative Commons Lizenz veröffentlicht. Die korrekte Lizenz finden Sie am Ende des Kapitels.

Ein Erratum zu diesem Kapitel ist verfügbar unter DOI 10.1007/978-3-658-12533-2_14

(C) SINUS Markt- und Sozialforschung GmbH 2016

M. Calmbach et al., Wie ticken Jugendliche 2016?, DOI 10.1007/978-3-658-12533-2_5 
Mobilität wird bis heute gerne mit Freiheit, Flexibilität und Unabhängigkeit assoziiert. Sie ist in diesem Verständnis Gradmesser für Modernität, wenn es darum geht, den heutigen Anforderungen an Beweglichkeit gerecht zu werden - sei es beruflich, familiär, geografisch, aber auch digital und letztlich mental. Der Philosoph Zygmunt Baumann bringt es wie folgt auf den Punkt: "Modern sein bedeutet, in Bewegung zu sein." Mobilität wird meist positiv gedacht. Sie kann Chancen eröffnen - insbesondere denjenigen, die über die notwendigen finanziellen, kulturellen und sozialen Ressourcen verfügen, um Mobilität angenehm und vor allem selbstbestimmt gestalten zu können.

Zunehmende eigenständige Mobilität ist auch integraler Bestandteil des Heranwachsens, angefangen vom Erlernen physischer Fortbewegungsmöglichkeiten (Krabbeln, Laufen) während der Kleinkindphase über das Nutzen erster Fahrzeuge (Dreirad, Fahrrad) sowie der selbständigen Orientierung im Straßenverkehr während der Kindheit bis hin zu motorisierten Verkehrsmitteln im öffentlichen Personenverkehr (ÖPV) oder in Privatnutzung (Motorrad, Pkw).

Gerade für Jugendliche bedeutet Mobilität auch eine Loslösung von den Eltern und die Möglichkeit, die eigene Alltagsgestaltung stärker selbst zu übernehmen, buchstäblich den eigenen Lebensweg zu wählen. Dabei ist die Abwägung verschiedener Mobilitätsalternativen jedoch neben persönlichen Vorlieben i.d. Regel noch geprägt von begrenzten Finanzmitteln und der Zustimmung der Eltern, v. a. wenn es um deren „Fahrdienste" geht. Naheliegend ist die Annahme, dass dies insbesondere für Jugendliche in ländlichen Regionen gilt, in denen der öffentliche Personenverkehr weniger gut ausgebaut ist.

Das folgende Kapitel zeichnet nach, welche Mobilitätsentscheidungen junge Menschen heute treffen bzw. in Zukunft treffen wollen und auf welche Herausforderungen sie dabei stoßen. Wir haben deshalb zunächst ermittelt, wie junge Menschen Mobilität allgemein erleben und wie sie das Unterwegssein im Alltag wahrnehmen. Daneben war es Aufgabe der vorliegenden Studie herauszufinden, wie die heute 14- bis 17-Jährigen ihren Mobilitätsalltag bewältigen, welche Verkehrsmittel sie nutzen und wie sie diese bewerten. Von Interesse ist dabei auch, ob sie Verkehrsmittel 
pragmatisch und situationsabhängig auswählen und mehrere Fortbewegungsmittel kombinieren (Stichwort "Multimodalität").

Wir haben die Jugendlichen auch gefragt, wie sie den öffentlichen Personenverkehr erleben, ob sie den Führerschein machen wollen und die Anschaffung eines PKW planen, was sie über Carsharing denken und schließlich auch, was für sie das ideale Verkehrsmittel ist.

Neben der Digitalisierung des Alltags sind auch Veränderungen wie die Verlängerung von Ausbildungszeiten und das Hinauszögern der Familiengründung wichtige Einflussfaktoren bzw. Rahmenbedingungen des Mobilitätsverhaltens junger Menschen. Vor diesem Hintergrund war es folgerichtig in der vorliegenden Untersuchung das Mobilitätsverhalten der 14- bis 17-Jährigen nicht isoliert, sondern im größeren Kontext ihrer lebensweltlich geprägten Gewohnheiten, Wünsche, Ziele und Werte zu betrachten. Besondere Aufmerksamkeit gilt hier der Frage, wie Jugendliche die zunehmenden Mobilitätsangebote im Hinblick auf ihre berufliche Zukunft im Kontext ihrer gesamten Lebensplanung wahrnehmen und bewerten.

Ohne Zweifel haben die in den letzten Jahren stattgefundenen Entwicklungen im Bereich der Informations- und Kommunikationstechnologie Auswirkungen auf das Mobilitätsverhalten vor allem der jungen Menschen. Dass die Bereitschaft zu physischer Mobilität als Folge der Digitalisierung generell zurückgeht, muss daraus nicht zwangsläufig folgen. Eine andere mögliche Konsequenz wäre die Öffnung des Horizonts vieler Jugendlicher für Vorgänge in anderen Teilen der Welt - also sozusagen eine Zunahme ihrer "mentalen Mobilität". Wie groß dabei in den verschiedenen Lebenswelten die Bereitschaft und der Wunsch sind, andere Teile der Welt heute und in Zukunft über Reisen zu entdecken, untersucht der vorletzte Teil dieses Kapitels.

Im Hinblick auf neue Technologien wird gerne unterstellt, dass junge Menschen per se über höhere technische Kompetenzen verfügen, Neuerungen in diesem Bereich unvoreingenommener und schneller annehmen und wie selbstverständlich in ihren Alltag integrieren. Studien zu den digitalen Lebenswelten junger Menschen haben jedoch schon gezeigt, dass es hier große Unterschiede innerhalb der Altersgruppe gibt, z. B. im 
Hinblick auf die Wahrnehmung möglicher Risiken und Nachteile. Daher widmet sich das folgende Kapitel abschließend auch der Frage, wie in den jugendlichen Lebenswelten Zukunftstechnologien im Bereich Mobilität, konkret selbstfahrende Autos, aufgenommen werden.

\subsection{Mobilitätsradius Jugendlicher}

\section{Vor allem Jugendliche aus postmodernen Lebenswelten möchten ihren Mobilitätsradius erweitern.}

In der Regel bewegen sich Jugendliche in ihrem Alltag auf vertrauten Strecken. Das ist so in allen Lebenswelten und trifft für Jugendliche auf dem Land gleichermaßen zu wie für Jugendliche in der Stadt. Lebensweltliche Unterschiede gibt es aber in der Bewertung. Typisch für die Jugendlichen aus dem postmodernen Segment (v. a. für Expeditive) ist, dass sie über ihre Mobilitätsgewohnheiten reflektieren und sich Gedanken machen, wie sie sich in Zukunft auf neuen Wegen orientieren müssen bzw. können. Viele freuen sich bereits darauf, später in eine andere Stadt zu ziehen, zu reisen und auf diese Weise ihren persönlichen Horizont zu erweitern.

Typische Zitate zur |llustration
Ich möchte später zumindest zeitweise weg aus $\mathrm{H}$. Ich finde es wichtig, die eigene Komfortzone zu verlassen, (...) dass ich später mal in eine andere Stadt gehe und dort die Möglichkeit habe, neue Wege zu laufen. (...) Ich gehe eigentlich immer zu den gleichen Orten und weiß genau, wo ich lang muss. Ich laufe auch immer den selben Weg und keinen anderen. Das ist alles im Alltag so drin. (weiblich, 15 Jahre, Stadt, Expeditive)

(7) Mir ist es auch wichtig, dass ich das Gefühl habe, dass ich nicht nur in meinem Dorf sitze und nicht über den Tellerrand rausschauen kann. Ich genieße es auch, die Leute zu sehen, wenn sie anders sind. Dass man auch sieht, das gefällt mir da besser oder das gefällt mir da nicht so gut. Einfach mal was sehen. (weiblich, 17 Jahre, Land, Expeditive) 
(1) Ja, Welt erkunden, Freiheit ein bisschen, Selbstentfaltung, also ja, neue Kulturen entdecken, neue Leute entdecken, alles neu kennenlernen, neue Eindrücke sammeln. (männlich, 17 Jahre, Stadt, Experimentalistische Hedonisten)

I Ich liebe es, Bahn zu fahren. Ich liebe es, Fahrrad zu fahren, ich liebe alles mit Unterwegs-Sein. (weiblich, 17 Jahre, Stadt, Experimentalistische Hedonisten)

Jugendliche aus dem traditionellen Segment sind hier hingegen zurückhaltender. Sie schätzen ihre "Komfortzone". Das spiegelt sich auch in ihren Reisewünschen wider, wie das folgende Kapitel zeigt.

(Draußen zu sein, klar. Aber den ganzen Tag weg zu sein, so lange wie möglich außer Haus sein, ist irgendwie nicht so mein Ding. (männlich, 17 Jahre, Stadt, Konservativ-Bürgerliche)

(1) Ich bin nicht gerne im Ausland unterwegs. Wegen der Sprache und anderen Sachen. (männlich, 15 Jahre, Stadt, Konservativ-Bürgerliche)

( Ich mag es auch nicht, in den Ferien irgendwo hinzufahren weit weg. Ich bin lieber zu Hause oder draußen ein bisschen. Ich muss jetzt nicht jeden Tag irgendwo sein und jeden Tag irgendwo anders Zeit verbringen. Es reicht mir, halt mal rauszugehen oder hier zu bleiben. Es wäre ein Stress, wenn man jetzt jede Woche von den Ferien zum Beispiel ausfüllen würde. Ich bleibe lieber zu Hause. (männlich, 14 Jahre, Stadt, Konservativ-Bürgerliche)

Bezüglich der im Alltag regelmäßig in Kauf zu nehmenden Fahrtzeiten, sind kaum Unterschiede zwischen Jugendlichen in der Stadt und auf dem Land zu erkennen. Auch Jugendliche in den Städten müssen durchaus einen längeren Schulweg zurücklegen oder weitere Entfernungen überbrücken, wenn sie Freundinnen und Freunde in anderen Stadtteilen besuchen wollen.

Die Freundin, die am nächsten wohnt, wohnt gleich zwei Häuser weiter. Und die andere Freundin wohnt in D. Das sind so zehn Kilometer von hier. Wir treffen uns aber meistens auf der Hälfte. Da kommt sie mit 
dem Fahrrad und ich auch. (weiblich, 15 Jahre, Stadt, Materialistische Hedonisten)

( Die weiteste Strecke sind immer diese 25 Kilometer zu meinem Freund. Das ist fast wie eine Fernbeziehung, wirklich. (weiblich, 17 Jahre, Stadt, Experimentalistische Hedonisten)

( Wenn ich zu Freunden fahre, dann fahre ich halt schon mal anderthalb Stunden, wenn ich jetzt mal nach L. muss oder so, mit der Bahn, was auch manchmal echt nervig ist. Ich meine, man ist ja voll schnell in $\mathrm{H}$. Ja, keine Ahnung, ich fahre meistens so zwischen einer halben Stunde und einer Stunde irgendwo hin. Geht eigentlich. (weiblich, 17 Jahre, Land, Sozialökologische)

\subsection{Fortbewegung im Alltag}

Wenig überraschend, sind die alltäglichen Hauptverkehrsmittel der Jugendlichen in erster Linie Bus und Bahn, das Fahrrad sowie "Chauffeur-Dienste" der Eltern. Dabei gibt es keine lebensweltlichen Unterschiede. Das eigene Rad ist beliebt, weil man flexibel ist und Kosten für Tickets spart. Mit Mietfahrrädern haben bislang nur wenige Jugendliche Erfahrungen gemacht. Altersbedingt stehen ihnen die Angebote auch noch nicht frei zur Verfügung, da die Registrierung bei den Anbietern in der Regel erst mit der unbeschränkten Geschäftsfähigkeit ab dem vollendeten 18. Lebensjahr möglich ist. Auf Taxis greifen „in Ausnahmefällen" lediglich die älteren Teenager am Abend bzw. in der Nacht an den Wochenenden zurück. Vereinzelt sind auch Skateboards und Longboards beliebte Fortbewegungsmittel, v. a. bei den hedonistischen Jugendlichen.

Typische

Zitate zur

Illustration
Eigentlich nur Bus und ab und zu Bahn, wenn ich in die Stadt fahre. So viele Verkehrsmittel benutze ich jetzt nicht. Fahrrad zur Schule meistens, weil es schneller geht. (männlich, 14 Jahre, Stadt, Adaptiv-Pragmatische)

Also ich komme mit dem Fahrrad und dem Bus überall hin. (männlich, 14 Jahre, Land, Konservativ-Bürgerliche) 
(1) Ich fahre eigentlich immer Fahrrad, auch wenn es regnet oder schneit. Es ist einfach das Schnellste. Zur Schule könnte ich auch zu Fuß gehen. Das würde dann fünf statt zwei Minuten dauern. Aber Fahrradfahren ist nicht so anstrengend, und es geht so schnell. Und es ist vor allem kostenlos. Denn die öffentlichen Verkehrsmittel sind so teuer geworden. Ich nutze sie natürlich auch mal, wenn ich ausgehe und das Fahrrad nicht so praktisch ist. Aber tendenziell nutze ich eher das Fahrrad. (weiblich, 17 Jahre, Stadt, Adaptiv-Pragmatische)

( Mit dem Fahrrad ist man halt viel flexibler. Auf die Bahn muss man immer warten, vor allem nachts. Würde ich niemals in Kauf nehmen, da irgendwie zwei Stunden zu warten. (männlich, 17 Jahre, Stadt, Adaptiv-Pragmatische)

(7) Mit dem Fahrrad fahre ich jetzt selten, weil jetzt ist wieder das Longboard da. (weiblich, 14 Jahre, Stadt, Experimentalistische Hedonisten)

Weil der Mobilitätsradius der Jugendlichen noch sehr begrenzt ist, spielen Mobilitätsdienste für längere Strecken (wie beispielsweise Mitfahrgelegenheiten) noch keine Rolle im Alltag. Auch Fahrten per Anhalter, sei es auf langen oder kurzen Strecken, sind nur für die wenigsten ein Thema. In den ländlichen Regionen werden auch "Mikrodienste" (beispielsweise Bürgerbusse oder Ruftaxis) nur vereinzelt genutzt, aber von Jugendlichen recht häufig als Verbesserungsvorschlag für das lokale Verkehrsangebot angeführt.

Der Moped-Führerschein hat für Jugendliche keine hohe Relevanz. Er stellt vor allem eine zusätzliche finanzielle Herausforderung dar, da der PKW-Führerschein in diesem Alter schon greifbar und für die meisten Befragten ein naheliegendes Ziel ist. Lediglich Einzelfälle auf dem Land berichten, wegen der sehr schlechten Verbindungen in jedem Fall den Moped-Führerschein machen zu wollen.

(7) Viele Freunde von mir haben ein Mofa oder einen Roller, aber ich wollte das gar nicht, weil ich finde es eh unnötig, wenn man dann auch mit 17 schon einen Autoführerschein machen kann, dann mache ich lieber einen Autoführerschein und dann kann ich mit 18 auch so einen kleinen Roller fahren. Deswegen fand ich es unnötig. Deswegen wollte ich es nicht machen. (weiblich, 17 Jahre, Land, Materialistische Hedonisten) 


\section{3 Öffentlicher Personenverkehr: Zufriedenheit und Image}

\section{Öffentliche Verkehrsmittel sind für manche mehr als Beförderungsmittel.}

Im Rahmen der vorliegenden Studie wurde den Jugendlichen die Frage gestellt, welches für sie das ideale Verkehrsmittel ist. Neben dem Auto, dem Fahrrad und kleinen Utopien wie einem Teleporter oder Multifunktionsfahrzeug werden hier auch die öffentlichen Verkehrsmittel genannt. In ihren Begründungen nennen die Jugendlichen dabei neben Flexibilität, Zuverlässigkeit, Schnelligkeit oder überhaupt der Möglichkeit, von A nach B zu kommen, folgende positive Aspekte:

- Menschen beobachten, in sozialen Austausch treten, alte Freunde wiedertreffen oder neue Kontakte knüpfen können,

- etwas von der Umgebung bzw. der Stadt sehen können,

- Zeit für sich haben, zum Lesen, Musik hören oder Nachdenken.

Typische Zitate zur Illustration
Ja total, ich liebe es, Bahn zu fahren. Ich weiß nicht, warum, aber ich liebe es, die Menschen zu beobachten. Ich liebe es, Zeit für mich zu haben und was von der Stadt zu sehen so nebenbei. (weiblich, 17 Jahre, Stadt, Experimentalistische Hedonisten)

(7) Also, ich finde sie eigentlich relativ cool, weil es ist auch so eine Chance, neue Leute kennenzulernen. Auch jetzt als der Bus nicht kam, habe ich so eine Portugiesin aus H. kennengelernt. (weiblich, 16 Jahre, Land, Sozialökologische)

Am liebsten fahre ich mit der Straßenbahn, weil ich es schön finde, dass man durch die Straßen fahren kann, aber man das Gefühl hat, man sitzt in einer Bahn. Man fährt halt durch die Stadt und kann sich alles angucken. (weiblich, 17 Jahre, Stadt, Materialistische Hedonisten) 


\section{Im Großen und Ganzen ist man mit Bus und Bahn zufrieden - Ausnahmen bestätigen die Regel.}

Unabhängig von Lebenswelt und Wohnortgröße ist die Pünktlichkeit von Bussen und Bahnen der wichtigste Aspekt bei der Bewertung des öffentlichen Personenverkehrs. Das gilt v. a. für den Schulweg: Man möchte morgens nicht früher aufstehen müssen, weil man mit Verspätungen bei Bus, Bahn usw. zu rechnen hat - sei es wegen hohem Verkehrsaufkommen, Baustellen oder Streiks. In Sachen Pünktlichkeit stellt man den öffentlichen Verkehrsunternehmen aber ein recht gutes Zeugnis aus. Manche finden lobende Worte, wenn es beispielsweise um die hohe Taktung der Verbindungen (vor allem in der Stadt) oder auch die Freundlichkeit des Fahrpersonals geht.

( Ich hatte mal einen Busfahrer, der hat allen einen guten Morgen gewünscht. So etwas fände ich toll. Da waren alle irgendwie gleich gut gelaunt. Er hat immer gesagt "An alle Fahrgäste, die gerade dazugestiegen sind: Herzlich wilkommen!" Das war total süß. Er hat auch immer zwischendurch, wenn mal Stau war, eine Durchsage gemacht und sich dafür entschuldigt. (weiblich, 17 Jahre, Stadt, Konservativ-Bürgerliche)

(1) Die Tram und so, das ist eigentlich schon alles pünktlich. Das gefällt mir. (männlich, 16 Jahre, Stadt, Materialistische Hedonisten)

( Ja, ich finde das Angebot hier sogar ziemlich gut, weil die fahren ja alle zehn Minuten, außer ab 20 Uhr zweimal in der Stunde. (weiblich, 17 Jahre, Stadt, Sozialökologische)

( Ich bin relativ zufrieden, zum großen Teil. Also, wenn ich jetzt zum Beispiel manchmal zu Freunden in einem anderen Dorf fahre, wo keine S-Bahn-Stelle ist, dann ist das halt schon doof, weil da kommt man nur mit einem Bus hin, der ungefähr alle zwei Stunden einmal fährt und am Wochenende gar nicht, und das ist halt echt blöd. Aber jetzt so zum Beispiel in den Bereichen, wo halt die S-Bahnen fahren, da ist das eigentlich voll cool. (weiblich, 17 Jahre, Land, Adaptiv-Pragmatische) 
Gleichwohl haben viele Jugendliche auch schon negative Erfahrungen, vor allem mit Verspätungen, gemacht.

Typische

Zitate zur

Illustration

( Der Bus, der jetzt hier vorbeifährt, kommt ungefähr jedes Mal zwölf Minuten zu spät. (männlich, 15 Jahre, Materialistische Hedonisten, Stadt)

( Manchmal haben die Verspätungen. Und das ist halt blöd, wenn man irgendwie einen Termin hat oder so. Aber man sollte ja zufrieden sein, dass die einen irgendwohin bringen. (männlich, 16 Jahre, Prekäre, Stadt)

( Und dafür, dass die Bahn oft unpünktlich ist oder es oft irgendwelche Baustellen gibt, finde ich die Preise total überteuert. (weiblich, 15 Jahre, Expeditive, Stadt)

( Der Bus, also ich würde ja eigentlich mit dem Bus von hier zur Bahn fahren, aber der fährt morgens, der ist immer so voll, dass der einfach vorbeifährt oft. Das heißt, manchmal kommt man gar nicht in die Schule und das ist richtig dumm. Mit dem Bus habe ich ziemlich schlechte Erfahrungen. Der kommt dauernd zu spät, manchmal gar nicht. Aber Bahn ist ganz gut. (männlich, 15 Jahre, Land, Adaptiv-Pragmatische)

Die erlebte Abhängigkeit vom öffentlichen Personenverkehr provoziert im Alterssegment von 14 bis 17 Jahren nicht selten Kritik - insbesondere, wenn sich die Jugendlichen als Kunden nicht ernst genommen oder schlecht behandelt fühlen.

\section{Neben den Fahrplanabweichungen werden folgende Punkte kritisiert:}

- Das Personal (Fahrer, Kontrolleure) wird nicht selten als unfreundlich erlebt.

- In den Verkehrsmitteln sei es oft heiß und stickig und gerade zu den Stoßzeiten (insbesondere in den Städten) auch zu eng.

- Die Sauberkeit in den Verkehrsmitteln lasse zu wünschen übrig (dreckige Sitze, Kaugummis oder anderer Müll) - vor allem die Mädchen bemängeln unhygienische Zustände. 
- Das Design der Verkehrsmittel (z. B. Innenausstattung) wird als wenig zeitgemäß und langweilig wahrgenommen.

- Es gebe kein (freies) WLAN in den Verkehrsmitteln.

(7) Viele Bahnfahrer sind unfreundlich. Die schreien dann einen an, und das kann ich gar nicht leiden. (männlich, 15 Jahre, Stadt, Adaptiv-Pragmatische)

Typische

Zitate zur

Illustration

Vielleicht bezahlen sie auch ihr Personal zu schlecht, ich weiß ja nicht, warum die immer so unzufrieden sind oder so unzufrieden rüber kommen. (weiblich, 16 Jahre, Stadt, Experimentalistische Hedonisten)

( ICh fahre generell nicht gern mit den Öffentlichen. Das liegt aber an den Leuten und nicht am Verkehrsmittel. In den alten 5er-Bussen, die derzeit eingesetzt werden, geht aber ab und zu die Klimaanlage nicht. Das ist echt ekelhaft. Man klebt an den Sitzen und an den Menschen. Im Prinzip ist es eine rollende Sauna. Das ist ekelhaft. Da läuft man lieber anstatt in diesem Bus zu hocken. (weiblich, 16 Jahre, Land, Experimentalistische Hedonisten)

(7) Es müsste sauberer sein in Bus und Bahn. Und es sollte mehr Sitzmöglichkeiten geben. Und die Busfahrer sollten netter sein (weiblich, 14 Jahre, Stadt, Expeditive)

( Die könnten die Züge ein bisschen moderner machen. (männlich, 15 Jahre, Stadt, Materialistische Hedonisten)

(1) Ich weiß nicht, Bahn und Busse sind immer dieselben, langweilig, nie fantasievoll, halt das Aussehen. (weiblich, 14 Jahre, Stadt, Adaptiv-Pragmatische)

( Es ist einfach, dass Züge ausfallen, dass Züge dann nicht pünktlich kommen, dass es zu warm ist in den Zügen. Und was mich morgens immer nervt, ich stehe am Bahnhof und die Ansage kommt, der Zug fältt leider aus oder hat Verspätung. Das muss jetzt echt nicht sein. Vor allem, wenn man spät dran ist und zur Schule muss und dann auch noch der Zug ausfällt. Die Lehrer sagen auch, man muss eigentlich so losfahren, dass man einen früher kommt, aber wenn ich dann um halb 
acht in der Schule bin, sehe ich es auch nicht ein, so früh da loszufahren. (weiblich, 17 Jahre, Stadt, Adaptiv-Pragmatische)

Von den Verkehrsunternehmen im Nah- und Regionalverkehr wünscht man sich entsprechend, dass sie zu allererst diese Mängel beheben. In Summe fällt das Image des öffentlichen Verkehrs bei Jugendlichen in ländlichen Räumen besser aus als bei Jugendlichen in Städten.

\section{Auf dem Land Wunsch nach besserer Anbindung an den öffentlichen Personenverkehr.}

Jugendliche auf dem Land bemängeln dagegen deutlicher als Städter eine unzureichende Abstimmung zwischen Schulzeiten und Fahrplänen. Sie wünschen sich bessere Verbindungen zwischen kleineren Ortschaften und einen Ausbau der Wochenend- und Abendfahrpläne. Forderungen nach einer besseren Anbindung werden vor allem von Jugendlichen aus bildungsnahen Lebenswelten artikuliert.

Typische

Zitate zur Illustration
Nur, was ich wirklich richtig schade finde, die Linie 5, die ja auch meine Linie ist, die fährt, glaube ich, ab 02:00 Uhr nachts überhaupt nicht mehr. Und das bis 04:00 Uhr morgens. (...) Und das kann doch nicht sein, dass die größte Strecke nicht mehr fährt. Es kann nicht sein, dass man sich dann extra ein Ruftaxi holen oder mit dem Fahrrad durch die Gegend gurken muss. (weiblich, 16 Jahre, Land, Experimentalistische Hedonisten)

Natürlich gibt es so Vorurteile, dass da immer nur Schüler mitfahren und irgendwelche Unterschichtsleute, aber das finde ich totalen Quatsch. Ich finde es eigentlich gut, wenn man die benutzt, gerade wenn man in der Stadt ist, ist es eigentlich viel praktischer. Nicht nur, dass man die Umwelt schont, sondern auch muss man nicht im Stau stehen oder so. Aber bei uns im Dorf ist es halt wirklich das Problem, dass nach acht Uhr kein Bus mehr fährt und sonst jede Stunde, da muss man sich schon sehr nach richten, das ist ein bisschen lästig. (weiblich, 17 Jahre, Land, Expeditive) 
(7) Nur ein Bus am Tag. Ich habe mir auch schon mehrfach Gedanken gemacht, vielleicht dort mal anzurufen, ob die nicht mehrmals einen Bus fahren lassen können. (männlich, 15 Jahre, Land, Experimentalistische Hedonisten)

( Dass mehr Orte einen S-Bahn-Anschluss bekommen, wünsche ich mir. Denn manche Orte erreicht man nur schlecht mit dem Bus, oder auch noch nicht mal mit dem Bus. Und manchmal fahren die Busse nur stündlich. Das ist dann immer schwer zu planen. (männlich, 15 Jahre, Land, Sozialökologische)

\section{Ängste vor unangenehmen Begegnungen im öffentlichen Personenverkehr, vor allem in den Städten.}

Die befragten Teenager sehen ihre Sicherheit in Bus und Bahn, vor allem in größeren Städten, nicht immer gegeben. Kriminalität oder bedrohliche Situationen werden nicht als generelles Problem gesehen, dennoch berichten vor allem Jugendliche aus den Städten von unangenehmen Begegnungen mit Obdachlosen, alkoholisierten oder unter Drogen stehenden Personen in den Bahnhöfen und Fahrzeugen. Insbesondere Mädchen fühlen sich nachts in Großstädten im öffentlichen Personenverkehr nicht immer sicher.

(7) Bei der U-Bahn kommt es drauf an, wo. Zum Beispiel xy-Straße oder so, da habe ich schon Angst, weil da sind voll ... Also manchmal sind da so alkoholisierte Leute. So Leute, die dich einfach ansprechen. (weiblich, 14 Jahre, Stadt, Materialistische Hedonisten)

(7) Naja, mit der U-Bahn eigentlich nicht unbedingt, weil da viele Leute sind, die einen anbaggern und eklig sind, oder irgendwelche Penner oder unseriöse Gestalten. Deswegen fahre ich eigentlich immer ganz gerne Bus. (...) Ich glaube, also am liebsten fahre ich eigentlich nachts oder abends mit dem Fahrrad nach Hause. Da fühle ich mich am sichersten und kann schnell wegfahren. (weiblich, 16 Jahre, Stadt, Sozialökologische) 
( Also gerade in den U-Bahnhöfen fände ich es gut, wenn da vielleicht einer sitzt, der wirklich anwesend ist. Nicht wirklich irgendwelche Kameragerätschaften, sondern wirklich ein Mensch, der das alles überprüft, wie es da zugeht und der auch Verstärkung schnell holen kann. [INT: IST DiR oder DeINEN FREUNDEN SCHON MAL WAS PASSIERT?] Nein, nicht wirklich. Also klar, unangenehme Anbaggereien, die dann auch mal übergriffig wurden, aber nie wirklich was Ernstes. (weiblich, 16 Jahre, Stadt, Sozialökologische)

\section{Umweltaspekte werden kaum thematisiert.}

Dass sich die Nutzung von Bussen und Bahnen durch eine höhere Umweltverträglichkeit (im Vergleich beispielsweise zum Autofahren) auszeichnet, wird von den Jugendlichen kaum thematisiert. Das bedeutet aber nicht, das Umweltschutz beim Thema Mobilität keine Rolle spielt. In den Diskussionen um die Anschaffung eines PKW wird dieser Aspekt sehr wohl genannt. In Bezug auf Bus und Bahn ist jedoch die Abhängigkeit der Jugendlichen zu groß, als dass die Schonung der Umwelt für sie bereits ein relevantes Nutzungskriterium sein könnte. Alternativen wie der eigene PKW stehen ihnen aktuell eben noch nicht zur Verfügung, so dass Busse und Bahnen im Vergleich zu anderen Fortbewegungsmitteln (zu Fuß, Fahrrad) im Hinblick auf die Umweltverträglichkeit keine Vorteile bieten.

\section{Der Fernverkehr hat ein überwiegend gutes Image.}

Vermutlich, weil die Altersgruppe den Fernverkehr mit der Bahn eher selten nutzt und in diesem Zusammenhang auch weniger schlechte Erfahrungen gemacht hat, fällt das Image des Fernverkehrs bei Jugendlichen aller Lebenswelten eindeutig gut aus - obwohl vielen durchaus präsent ist, dass auch der Fernverkehr von Verspätungen und Ausfällen betroffen ist und die Preise relativ hoch sind. Den Angeboten des Fernverkehrs schreibt man aber insbesondere einen hohen Komfort (WLAN, saubere Verkehrsmittel) und guten Service (Bordbistros, Toiletten) zu. 
Interessant ist auch, dass Fernlinienbusse bei der Altersgruppe nur vereinzelt mit Fernverkehr in Verbindung gebracht werden.

(8) Naja, Fernverkehr; da sind halt die Züge meistens ein bisschen voller, aber sind dafür deutlich besser ausgestattet, angenehmere Sitzmöglichkeiten. (männlich, 16 Jahre, Land, Adaptiv-Pragmatische)

Typische Zitate zur Illustration

( Die Züge der Bahn finde ich cool. Die sehen modern aus, sind modern, ist eine ordentliche Klimaanlage drin, man fühlt keine Unebenheiten beim Fahren, sind schnell. (männlich, 14 Jahre, Stadt, Adaptiv-Pragmatische)

(1) Am liebsten fahre ich ICE, weil ich den toll finde, weil dieser Zug schnell ist und innen schön. (weiblich, 17 Jahre, Stadt, Materialistische Hedonisten)

(7) Also ich fahre ja mit dem Zug nach Mainz, zu meinem Papa, in Fernverkehrszüge sind die Sitze größer, man hat mehr Beinfreiheit. (männlich, 15 Jahre, Adaptiv-Pragmatische, Land)

( Im Zug ist es gemütlicher und du hast eine Steckdose für den Laptop, Smartphone, was auch immer. (weiblich, 14 Jahre, Land, Konservativ-Bürgerliche)

\section{Man greift oft auf Mobilitäts-Apps und Internetseiten der Mobilitätsunternehmen zu.}

Da Jugendliche im Alltag häufig vertraute Wege nutzen, verwundert es nicht, dass Apps von Mobilitätsanbietern zwar als sehr praktisch, aber nicht als unverzichtbar gelten. Man kennt die Fahrpläne für die wichtigsten Strecken in der Regel schlicht auswendig. Dennoch nutzen die 14- bis 17-Jährigen Fahrplan-Apps ganz selbstverständlich, wenn ein entsprechender Anlass vorliegt. Die expliziten Nicht-Nutzer von Apps informieren sich teilweise über die Homepages der Mobilitätsanbieter, fragen bei den Eltern nach oder nutzen die Fahrplanausdrucke an Haltestellen und in den Fahrzeugen. Lebensweltliche Unterschiede zeigen sich bei der App-Nutzung nicht. 
Typische Zitate zur |llustration

(7) Also die S-Bahn-Zeiten mache ich eigentlich immer aus dem Kopf, da fährt ja alle Viertelstunde eine, das ist dann nicht so schwer. Und wenn ich dann mal in die Stadt fahre, dann habe ich halt da so eine Stadtplan-App. (weiblich, 17 Jahre, Land, Materialistische Hedonisten)

( Ich gucke einfach auf den Fahrplan und dann merke ich mir das meistens. Ist ja auch eine Routinestrecke für mich, nach K. beispielsweise weiß ich ganz genau, 20:48 Uhr kommt dort die Bahn, muss ich nehmen, sonst muss ich warten. (männlich, 16 Jahre, Land, Adaptiv-Pragmatische)

( Es gibt ja diese Apps mit den Zeiten und da guck ich halt immer vorher, wie ich am besten nach Hause komme und wann ich so ungefähr losgehe, dass ich nicht eine Stunde irgendwo warten muss. (weiblich, 15 Jahre, Stadt, Materialistische Hedonisten)

8 Ja, wenn ich mit dem Bus fahren muss, wenn es wirklich weit weg ist, auf jeden Fall über easy.GO. (weiblich, 16 Jahre, Stadt, Experimentalistische Hedonisten)

( Über Fahrpläne oder Verbindungen informiere ich mich über die Deutsche Bahn App. Oder halt, wenn ich gerade an der Bushaltestelle stehe, gucke ich auf den Busplan. (weiblich, 17 Jahre, Land, Konservativ-Bürgerliche)

Als wichtiges Element moderner Mobilität gelten heute ganzheitliche MobilitätsApps, die sämtliche Angebote innerhalb einer Stadt vernetzen und die jeweils schnellste, einfachste und bei Bedarf kostengünstigste (oder auch umweltschonendste) Verbindung ermitteln. Besonders für die postmodernen Lebenswelten werden mit einem solchen Angebot zentrale Bedürfnisse erfüllt. Typischerweise berichten gerade Jugendliche aus diesem Segment, dass das ideale Verkehrsmittel für sie situationsabhängig wechselt - hier ist offenbar der Trend zu multimodaler Nutzung, wie ihn Verkehrsexperten für das Mobilitätsverhalten der Zukunft prognostizieren, bereits Realität. 


\subsection{Führerschein und PKW-Besitz}

\section{Vor allem pragmatische Gründe sprechen für den Führerschein bzw. ein eigenes Auto.}

Ein Trend hin zu einer geringeren PKW-Nutzung wird in Deutschland seit den 1990er Jahren beobachtet. Auch die Quote des Führerscheinerwerbs ist zurückgegangen, stagniert aber seit Jahren auf hohem Niveau. So äußern auch die allermeisten Befragten dieser Studie den Plan, den Führerschein machen zu wollen. Wenn Experten von einem Bedeutungsverlust des PKW in der jungen Generation sprechen, dann meint dies vor allem, dass andere Mobilitätsoptionen parallel zur PKW-Nutzung an Bedeutung gewonnen haben.

In den für diese Studie geführten Gesprächen zeigt sich, dass aus Sicht der heute 14- bis 17-Jährigen wenig gegen den Führerscheinerwerb und das Autofahren spricht. Noch am ehesten werden Umweltbelastungen durch den Autoverkehr und hohe Kosten als Probleme genannt ohne damit die grundsätzliche Akzeptanz des Autos in Frage zu stellen. Der Führerschein und später ein eigener Wagen gehören für die meisten Jugendlichen - unabhängig von Geschlecht, Wohnort(größe) und Lebenswelt - zum Erwachsenwerden quasi selbstverständlich dazu. Man blickt in der Regel optimistisch und mit Vorfreude auf die Verantwortung, die mit der eigenen Automobilität einhergeht.

Die Gründe, weshalb Jugendliche den Führerschein erwerben wollen und sich ein eigenes Auto wünschen, sind überwiegend praktischer Natur:

- Man möchte (jederzeit) von A nach B kommen, ohne dabei auf starre Fahrpläne der öffentlichen Verkehrsanbieter angewiesen zu sein.

- Insbesondere Jugendliche aus ländlichen Wohnorten meinen, mit dem Auto besser am Leben in der Region teilhaben zu können.

- Man geht davon aus, ohne Führerschein im Berufsleben benachteiligt zu sein.

- Man kann Freunde mitnehmen und Dinge besser transportieren, z. B. beim Einkaufen. 
Vor allem die Jugendlichen aus ländlichen Regionen sprechen viel über die Vorteile des Führerscheins bzw. eines eigenen Autos. Wer wann den Führerschein macht, ist ein wichtiges Thema im Alltagsdiskurs - nicht zuletzt, weil man sich ein "Fahrernetzwerk" aufbauen möchte, um selbst mobiler sein zu können. Einige blicken auch neidisch auf die etwas Älteren im Freundeskreis, die bereits die Freiheiten des Autofahrens genießen können. Für Jugendliche in der Stadt ist das Thema etwas weniger bedeutsam.

Typische Zitate zur Illustration
Man ist mobiler unterwegs und muss sich nicht mehr auf Bus und Bahn verlassen. Man ist eigenständiger. Das finde ich einen großen Schritt zur Eigenständigkeit, wenn man damit umgehen kann. (weiblich, 16 Jahre, Land, Adaptiv-Pragmatische)

(7) Mit einem Auto muss man dann nicht mehr sagen "Mama, kannst du mich abholen?" oder "Papa, kannst du mich da und da hinfahren?". Sondern ich sage dann einfach, ich will dahin und fahre dann dahin. (weiblich, 16 Jahre, Land, Adaptiv-Pragmatische)

( Mit Auto muss man nicht Leute fragen, ob sie einen da hinbringen können. Dann kann man selber fahren. (männlich, 15 Jahre, Stadt, Prekäre)

( Ja, auf jeden Fall. Erst mal mache ich ja meinen Führerschein. Und dann würde ich mir auch später ein Auto kaufen. (männlich, 16 Jahre, Stadt, Materialistische Hedonisten)

(7) Weil für den Job später, da kommt man eigentlich nicht weit ohne Führerschein. Und auch so Einkaufen, alles mögliche, ist mit dem Führerschein einfach auch sehr komfortabel. (männlich, 16 Jahre, Stadt, Materialistische Hedonisten)

( Ohne Auto kann man irgendwie nicht leben, finde ich. Man braucht irgendwie überall so ein Auto, um etwas zu transportieren oder irgendwo hinzufahren oder in Urlaub zu fahren. (weiblich, 14 Jahre, Stadt, Adaptiv-Pragmatische)

Sie finden alle den Führerschein wichtig. Da geht es auch um die Flexibilität. Es geht dabei auch um das Einkaufen. Man hat dann ein Transportmittel. Auch wenn man mit Freunden irgendwohin will, kann man 
sehr gut das Auto nutzen. Man kann auch ein bisschen weiter weg fahren und dort eine Nacht verbringen, um ein wenig zu feiern. (männlich, 16 Jahre, Land, Adaptiv-Pragmatische)

( Ja, also die hohen Kosten und Spritkosten. Das ist eben der Nachteil, den es gibt bei Autos. Eigentlich nur der Kostenfaktor, finde ich. Und eben für die Umwelt blöd, aber sonst ... (männlich, 16 Jahre, Land, Adaptiv-Pragmatische)

\section{Zu wenig Geld und zu wenig Zeit sind die zentralen Barrieren beim Führerscheinerwerb.}

Wann mit der Fahrschule begonnen wird, hängt zum einen davon ab, wann man das Geld dafür zusammen hat. Zum anderen spielt aber auch Zeit eine wichtige Rolle. Innerhalb der knappen Zeitbudgets zwischen Schule, Hausaufgaben, Lernen, Freizeitaktivitäten und Nebenjob bleibt oft nicht viel Zeit für die Fahrausbildung.

(1) Ich hatte erst überlegt, es diesen Sommer schon zu machen, aber es war dann einmal mit dem Geld - ich müsste einen Teil selber bezahlen - und dann das andere ist dann auch Zeit. Wenn ich jetzt regelmäßig wieder babysitten gehen sollte, dann kann ich auch nicht noch gleichzeitig meinen Führerschein machen, wenn ich den ganzen Tag unterwegs bin. Ich kann sowieso im Moment nicht fahren und würde im Moment auch nicht so viel fahren und dann würde ich es gerne im nächsten Sommer spätestens machen. (weiblich, 17 Jahre, Stadt, Adaptiv-Pragmatische)

( Ich will ihn aber erst nach dem Abitur machen, weil ich jetzt schon genug lernen muss. (weiblich, 17 Jahre, Stadt, Adaptiv-Pragmatische)

Spaß am Autofahren ist dabei kein dominantes Motiv und wird nur vereinzelt angesprochen - nicht zuletzt wohl auch, weil es nicht mehr als zeitgemäß gilt. Wirklich leidenschaftlich sprechen nur wenige Befragte über das Autofahren, vor allem Jungen mit Migrationshintergrund und aus unterschichtigen Lebenswelten. Sie verbinden mit dem Autofahren nicht nur Freiheit und Lebensfreude, sondern auch gesellschaftliche Teilhabe und Statusgewinn. 
Typische Zitate zur |llustration

( Ich weiß jetzt schon, ich werde Autofahren lieben. Ich finde Autos auch cool, das kann ein Renault Clio sein, mir egal, muss ja kein Lamborghini sein. Aber dann hat man so ein riesiges Freiheitsgefühl, man kann herumfahren, man ist schneller da und da, es ist einfach cool, wenn man ein Auto hat. (männlich, 15 Jahre, Stadt, Materialistische Hedonisten)

Ich interessiere mich sehr für schnelle Autos, also Sportwagen. Und ich gucke mir auch gerne auf YouTube "GRIP - Das Motormagazin" und "Die PS-Profis" an, damit ich ein paar Details zu den Autos kennenlerne. Es interessiert mich halt schon sehr, was die für Motoren haben und alles. (männlich, 15 Jahre, Stadt, Materialistische Hedonisten)

( Wir haben Autorennen gemacht. Gedriftet durch ganz L. Wir sind an einer Stelle losgefahren und jeder ist einen anderen Weg gefahren. Wir mussten dann zum Beispiel in N. am Hauptplatz ankommen. Wer als erster da ist hat 50 Euro gewonnen. Jeder musste 10 Euro dazulegen. (weiblich, 14 Jahre, Stadt, Prekäre)

8 Ach, mit Freunden einfach ein bisschen rumcruisen, so Musik hören dabei, und verschiedene Orte ... Du kannst halt irgendwo hinfahren und ran fahren und kurz abchecken und dann wieder losfahren. (männlich, 16 Jahre, Land, Adaptiv-Pragmatische)

Am wenigsten "Auto-minded" sind die Angehörigen der postmodernen Lebenswelten. Expeditive halten den Führerscheinerwerb aus pragmatischen Gründen meist für sinnvoll, verzichten aber gerne auf ein eigenes Auto, wenn es (wie etwa in den Großstädten) andere attraktive Mobilitätsangebote gibt.

\section{Ein eigenes Auto ist eher ein Fern- denn ein Nahziel.}

Die meisten Jugendlichen gehen davon aus, dass sie zumindest irgendwann einmal ein eigenes Auto besitzen wollen, denn erst durch das eigene Auto wird das Versprechen des Führerscheins auf Unabhängigkeit und Bequemlichkeit voll eingelöst. Lediglich in den postmodern geprägten Lebenswelten finden sich Hinweise darauf, dass ein Erwachsenenleben auch ohne eigenen PKW vorstellbar wäre. Die Anschaffung 
eines PKW ist allerdings für die meisten noch kaum greifbar, gilt es doch zunächst einmal, den Führerschein zu finanzieren. Und es ist den meisten völlig klar, dass das erste eigene Auto noch warten muss. Diese Situation wird aber lebensweltspezifisch teilweise unterschiedlich bewertet.

- Nicht nur Jugendliche aus dem postmodernen Segment, sondern auch Adaptiv-Pragmatische (der junge Mainstream) beschreiben es als völlig unproblematisch, zunächst kein eigenes Auto zu besitzen. Wenn sie beispielsweise an die kommende Studien- oder Ausbildungszeit denken, erscheint es utopisch, bereits für ein eigenes Auto Geld auszugeben. Für die meisten dieser Jugendlichen gehört ein eigenes Auto aber irgendwann zum Leben dazu - auch wenn mancher diejenigen bewundert, die ganz ohne Auto auskommen.

Wenn ich zum Beispiel studiere, brauch ich noch kein eigenes Auto. Man muss ja auch die Benzin- und Reparaturkosten zahlen. Das ist für einen Studenten etwas zu teuer. Aber irgendwann glaube ich schon, ja. (weiblich, 17 Jahre, Stadt, Adaptiv-Pragmatische)

( Wenn ich fertig mit Studieren bin und einen Job habe, auf jeden Fall. Aber ich glaube, als Student braucht man eigentlich kein Auto. Gerade wenn man in einer Großstadt studiert, hat man sein Studententicket und dann braucht man das nicht. Da fängt man eh nichts mit so einem Auto an. (weiblich, 16 Jahre, Land, Expeditive)

$>\quad$ Nein, das muss nicht sein. Nicht wirklich. Es geht auch mit den Öffentlichen. Und es gibt auch Leute, die schaffen alles nur mit dem Fahrrad. Respekt. (weiblich, 16 Jahre, Land, Expeditive)

- Bei Prekären und insbesondere Materialistischen Hedonisten spielt das erste eigene Auto hingegen eine wichtige Rolle. Man kann es in diesen Lebenswelten meist kaum abwarten, bis man endlich einen eigenen Wagen hat (das gilt insbesondere für die Jungen).

(7) Ja, so ein eigenes ist dann immer besser. (...) Zum Beispiel wenn ich eine Farbe von mattgrün will, halt so ein olivengrün, dann kann ich das 
halt auch selber wählen. Da muss keine andere Person halt dazu was sagen. (männlich, 16 Jahre, Stadt, Materialistische Hedonisten)

( Ich würde den Führerschein gern mit sechzehneinhalb machen, um mir dann gleich mit 16 oder 17 ein Moped zu holen oder irgendeine gedrosselte Maschine. Bis zu einer bestimmten Zahl darf man die ja fahren. Und mit 18 will ich dann gleich ein Auto. (männlich, 15 Jahre, Stadt, Materialistische Hedonisten)

(1) I bin dann nicht mehr auf meine Mutter angewiesen. Wenn ich zum Beispiel nachts um drei Hunger auf McDonalds habe, kann ich einfach fahren. Ich komme einfach überall hin damit. Ich kann machen, was ich will, und muss nicht immer Mama fragen. (weiblich, 15 Jahre, Stadt, Materialistische Hedonisten)

( Ich will mit 17 Führerschein machen und gleich ein Auto. (männlich, 15 Jahre, Stadt, Prekäre)

Es wird schon so ein bisschen angegeben, wer wann seinen Führerschein und das Auto bekommt. Es ist schon sehr wichtig in meinem Freundeskreis. (männlich, 15 Jahre, Stadt, Materialistische Hedonisten)

Tendenziell ist das eigene Auto für Jugendliche in ländlichen Regionen wichtiger als für Jugendliche in der Stadt. Viele der städtischen Jugendlichen können sich gut vorstellen, sich einen PKW mit den Eltern zu teilen. Sie sind auch für die Car-Sharing-Idee eher zu begeistern.

( Das brauche ich nicht unbedingt, da ich weiß, dass mein Vater mir das Auto geben würde, wenn ich es brauche, weil er auch nicht jeden Tag fährt, da ist es dann okay. Ich müsste wahrscheinlich dann den Sprit, den ich verbrauche, wenn ich dann tanken muss, selbst bezahlen. Ich bin nicht der Meinung, dass man unbedingt ein eigenes Auto braucht. (weiblich, 17 Jahre, Stadt, Adaptiv-Pragmatische)

(Jetzt am Anfang will ich noch keins eigentlich, wegen der Versicherung und allem. Aber dann kann ich es mir eigentlich schon vorstellen, so ein gebrauchtes halt ... Hauptsache es fährt. (weiblich, 17 Jahre, Land, Sozialökologische) 


\section{Das Auto ist in vielen Lebenswelten kein Statussymbol.}

Auch wenn die große Mehrheit der befragten Jugendlichen (später einmal) ein Auto haben will, heißt das nicht zwangsläufig, dass der PKW erste Priorität bei der Verkehrsmittelwahl hat. Auffällig ist, dass Jugendliche oft sehr nüchtern über das Auto sprechen: Hauptsache es fährt und sieht einigermaßen gut aus. Außerdem soll es im Verbrauch und von den Anschaffungskosten her günstig und nicht zu neu sein, damit man sich nicht über jeden Kratzer ärgern muss.

( Solange es fährt. Ich interessiere mich nicht wirklich für Autos. Ich kann auch die Marken nicht wirklich auseinanderhalten. (weiblich, 15 Jahre, Stadt, Expeditive)

Typische Zitate zur Illustration

(1) Ich habe einen Freund, der ist Autofanatiker. Aber ich will einfach fahren. Ich will jetzt nicht so gerne einen Mini haben, sondern ein ganz normales Auto wäre okay. (weiblich, 15 Jahre, Stadt, Konservativ-Bürgerliche)

( Ich glaube, es wäre ein Nutzfahrzeug. Ich würde schon eins kaufen, was mir auch gefällt und das ich cool finde. Aber im Vordergrund stände wahrscheinlich dann der Nutzen. (weiblich, 17 Jahre, Stadt, Adaptiv-Pragmatische)

(1) Es muss jetzt nicht irgendwie totaler Schrott sein, dass man gleich das Gefühl hat, es fällt auseinander, es muss aber jetzt auch kein Luxuswagen sein. Es soll halt gut fahren, man soll sich vielleicht auch sicher drin fühlen. Natürlich soll es auch ganz gut aussehen, so vom Design her, aber das ist jetzt nicht das Wichtige. (weiblich, 17 Jahre, Land, Expeditive)

Das Auto als Identifikationsobjekt und Statussymbol spielt überwiegend nur eine geringe Rolle. Für klassische Prestigeaspekte sind noch am ehesten Materialistische Hedonisten, Prekäre und Adaptiv-Pragmatische sensibel. 
Auszüge aus Hausaufgabenheften zur Frage: WAS GIBT DeINEM LeBEN SInN?

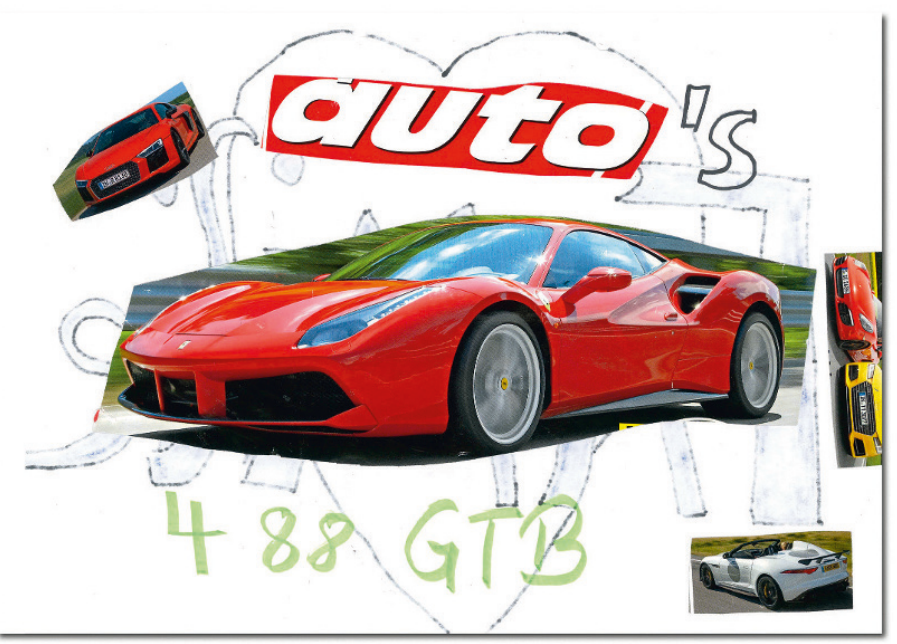

männlich, 15 Jahre, Stadt, Materialistische Hedonisten
Typische Zitate zur Illustration
(7) Ein BMW wäre cooler als irgendein Citroën. Aber es kommt drauf an, wie viel Geld ich dann haben werde. (männlich, 15 Jahre, Land, Adaptiv-Pragmatische)

Also das Aussehen ist schon wichtig. Dann die Anlage. Muss jetzt nicht besonders aussehen, aber einfach schick so. Also Audi sieht eigentlich gut aus, finde ich. Aber ist halt teuer. Deswegen wahrscheinlich eher so BMW. Aber kein neues Modell, das könnte ich mir wahrscheinlich nicht leisten. (männlich, 17 Jahre, Stadt, Adaptiv-Pragmatische)

(7) Einen Mercedes. Ich finde den schön. Und elegant sehen die auch aus. (weiblich, 15 Jahre, Stadt, Materialistische Hedonisten)

(7) Also, ein Golf ist ja geil. Vor allem ein Golf II, wenn er tiefergelegt oder in matt ist. (weiblich, 16 Jahre, Land, Materialistische Hedonisten)

(1) Ich liebe Mercedes. Also Mercedes ist einfach das beste Auto für mich. Sieht einfach geil aus. (männlich, 15 Jahre, Stadt, Prekäre) 
(1) Ich will natürlich bald ein eigenes Auto - ein sehr schönes Auto. Kann BMW sein. BMW ist eigentlich gut, oder? (weiblich, 15 Jahre, Stadt, Prekäre)

(7) Später ein Traumauto. Vielleicht ein Koenigsegg oder so was Krasses. Oder ein Pagani. (männlich, 15 Jahre, Stadt, Prekäre)

Allerdings kommt dem Auto als Lifestyle-Ausweis bzw. als ästhetisches Persönlichkeits-Statement - auch in gehobenen Lebenswelten - eine gewisse Bedeutung zu (neues Prestige).

( Ich finde Minis ziemlich cool. Man kann sich das auch als Cabrio kaufen, das finde ich ganz cool. Aber am liebsten hätte ich, wenn ich dann auch eine Familie habe, so einen ganz alten VW Bus. Nicht so die neuen, sondern diese ganzen alten, diese Hippie-Busse, die finde ich so richtig cool. (weiblich, 16 Jahre, Land, Expeditive)

Ich würde mein Auto auf jeden Fall pink machen. Aber nicht so ein knalliges Pink, sondern so ein lachsrosa eigentlich. Und dann vielleicht noch mit so Streifen oder irgend so ein schönes Auto. (weiblich, 14 Jahre, Land, Experimentalistische Hedonisten)

\section{Die idealen Verkehrsmittel sind für die Jugendlichen das Auto, Bus und Bahn sowie das Fahrrad.}

Bei der Frage nach dem idealen Verkehrsmittel werden häufig das Auto, Bus und Bahn und das Fahrrad genannt. Jedes Verkehrsmittel hat seine Vor- und Nachteile. Als „ideal“ wird oft pragmatisch das Verkehrsmittel genannt, das am besten in die derzeitige Lebenssituation der Jugendlichen passt. Die öffentlichen Verkehrsmittel werden aufgrund des niedrigen Preises (oder der schon vorhandenen Monatskarte) sowie der Reichweite und Verfügbarkeit bevorzugt. Vorteile des Fahrrads sind, dass es (fast) nichts kostet, nicht von einem Fahrplan abhängt und schneller sein kann, da es nicht anfällig für Staus und Streiks ist. Welches Verkehrsmittel am besten „passt“, hängt vom Reisezweck ab. Eine generelle Präferenz für das Auto ist noch am deutlichsten bei den Materialistischen 
Auszüge aus Hausaufgabenheften zur Frage: WAS GIBT DEINEM LeBEN SINN?

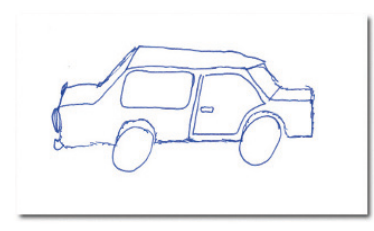

männlich, 15 Jahre,

Materialistische Hedonisten, Stadt

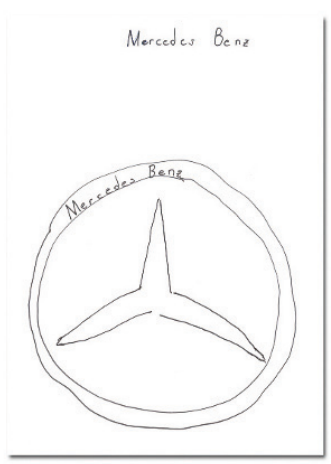

männlich, 15 Jahre,

Materialistische Hedonisten, Stadt

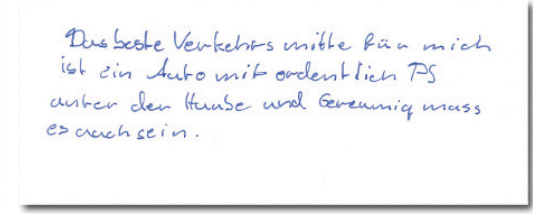

männlich, 15 Jahre,

Materialistische Hedonisten, Stadt

Mercedes Benz, Audi

männlich, 15 Jahre,

Materialistische Hedonisten, Stadt

Dus ideale Verkohismittel für mich ist dus Auto. Man komast sduell won A nach B, man kann Einkaute etc. mitnohmer und man ist nicht an gewisse Fahrieiter dobunden.

männlich, 16 Jahre,

Materialistische Hedonisten, Stadt

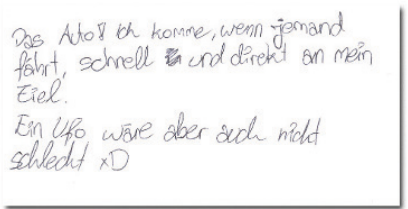

weiblich, 16 Jahre,

Materialistische Hedonisten, Land

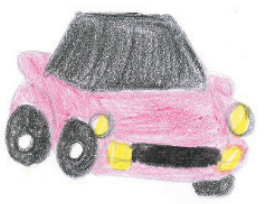

wenn ich mein Füherschein habe, denke ich das ich uberall mit dem Auto hinfahren werde. uit dem Auto ist man am schnellsten unterwegs.

weiblich, 16 Jahre,

Adaptiv-Pragmatische, Stadt

Hedonisten und Jugendlichen aus ländlichen Regionen erkennbar, auch wenn - kontrastierend zur aktuellen Abhängigkeit von öffentlichen Verkehrsmitteln - seine Vorzüge (Schnelligkeit, Flexibilität, Convenience, Spaßfaktor, Privatheit) eindrücklich beschrieben werden.

Bus und Bahn werden besonders von den Sozialökologischen und Expeditiven Jugendlichen als ideales Verkehrsmittel genannt (wenngleich diese Jugendlichen auch für andere Verkehrsträger offen sind).

Für lange Strecken bevorzugen die Jugendlichen das Flugzeug und Fernzüge, da diese Reisearten als besonders schnell und bequem gelten. Auch noch häufig wird das Auto genannt, da es flexibler ist. 
Auszüge aus Hausaufgabenheften zur Frage:

WAS IST FÜR DICH DAS IDEALE VERKEHRSMITTEL UND WAS BEDEUTET ES DIR?

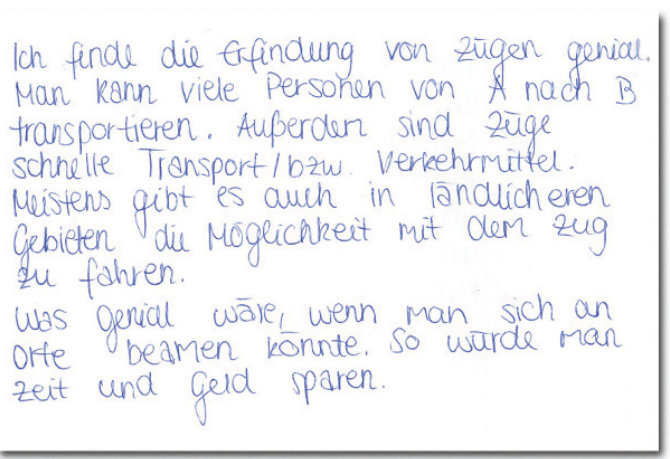

weiblich, 17 Jahre, Sozialökologische, Land

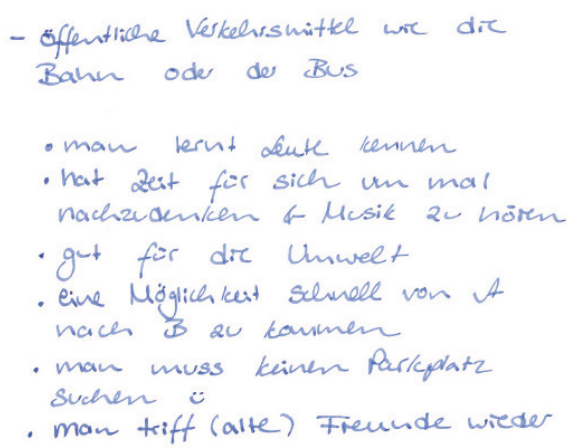

weiblich, 15 Jahre, Sozialökologische, Land

( Am bequemsten ist halt das Flugzeug, aber man kann auch nicht überall in Deutschland mit dem Flugzeug hin. Dann ist das Bequemste Typische das Auto, weil man auch selbstständig entscheiden kann, wann Zitate zur man losfährt und auch mobiler ist. (weiblich, 17 Jahre, Stadt, Illustration Adaptiv-Pragmatische)

$>$ Mit dem Flugzeug eigentlich. [INT: WAS FINDEST DU GUT DARAN?] Dass man halt eigentlich so schnell und eigentlich so gemütlich und entspannt von einem Ort zum anderen kommt, ohne halt irgendwie so lange im Auto sitzen zu müssen oder im Bus. Das ist halt schon, finde ich, angenehm. (weiblich, 17 Jahre, Land, Adaptiv-Pragmatische)

(7) Mit einer Bahn. Oder am besten wäre ein Flugzeug. Ich bin erst einmal geflogen, ich fand das ultracool. Aber ich finde Bahn auch sehr, sehr gemütlich. Ich mag Bahnfahren sehr. Mit dem Bus kann ich mir nicht so gut vorstellen, ich bin zwar schon lange, 23 Stunden mit dem Bus gefahren, aber das ist halt nicht so angenehm wie Bahn oder Flugzeug. (männlich, 15 Jahre, Stadt, Materialistische Hedonisten)

( Ich fahre richtig gern Zug. Ich fliege nicht so gern. Ich fahre eigentlich viel lieber Zug. Oder mit dem Auto ist es auch gut. Man ist dann 
viel flexibler. Mit dem Zug muss man ja immer die vorgegebene Strecke fahren. Andererseits ist es mit dem Zug entspannter, weil man sich nicht auf das Fahren konzentrieren muss. (weiblich, 17 Jahre, Stadt, Expeditive)

( Mit dem Zug. Ich finde Zugfahren schön. Solange der Zug nicht total krass überfüllt ist. Ich finde Zugfahren total entspannend. Man kann dabei raus schauen und muss sich nicht darum kümmern, wo der Zug hinfährt. Und man weiß, man ist etwa dann und dann da. Ich finde Zugfahren schön. Aber wenn ich den Führerschein hätte, würde ich lieber mit dem Auto fahren. Ich wäre dann unabhängiger. Es würde keine Rolle spielen, ob der Zug Verspätung hätte oder gar nicht kommt. Ich müsste dann auch keine Umwege durch Städte fahren, sondern könnte mit dem Auto direkt an mein Ziel fahren. Ich könnte auch jemanden mitnehmen, ohne dass es mehr kostet. Es ist ja der gleiche Preis, wenn ich alleine oder mit jemandem zusammen im Auto fahre. (weiblich, 15 Jahre, Stadt, Expeditive)

\subsection{Car-Sharing}

\section{Car-Sharing ist unter Jugendlichen noch ein Randthema, wird aber positiv bewertet.}

Von Car-Sharing haben vor allem die Jugendlichen in den Städten und die Gymnasiasten schon gehört, wenngleich sie das Konzept nicht immer näher erklären können. Spontan verstehen manche unter Car-Sharing Mitfahrgelegenheiten oder die Nutzung eines Autos gemeinsam mit Freunden und Bekannten.

Wenn ich das Wort Car-Sharing höre, stelle ich mir vor, dass man im Internet eine Plattform hat, wo man Leuten schreibt, dass man an einem bestimmten Tag zu einer bestimmten Zeit an einen bestimmten Ort fährt. Und dann können da einfach Leute mitfahren. Und da kann 
man sich die Benzinpreise teilen. Und man tut noch ein bisschen was für die Umwelt. (männlich, 15 Jahre, Land, Sozialökologische)

Unter den Haupt- und Realschülern ist Car-Sharing deutlich weniger bekannt. Generell haben nur wenige bislang den Dienst schon genutzt (z. B. über Eltern oder Freunde).

Die Idee einer organisierten gemeinschaftlichen Nutzung von Autos wird größtenteils positiv bewertet ( $v$. a. in den sozial gehobenen Lebenswelten), auch von denjenigen, die bisher noch nichts davon gehört haben. Gegen das Car-Sharing sprechen die dem Konzept eigenen Grenzen individueller Verfügbarkeit.

I Ich finde das eigentlich praktisch, obwohl es halt auch nicht so persönlich ist, weil wenn es dann gerade in Benutzung ist und nirgendwo eins in deiner Gegend ist, dann ist es trotzdem wieder nicht da. Es kann praktisch sein, auf jeden Fall, weil es dann doch nicht so viel kostet, wie es selber zu bezahlen, aber es ist halt trotzdem auch nicht deins. Du kannst nicht irgendwie Taschen im Auto liegen lassen, wenn du das nächste Mal einkaufen gehst oder so, und das finde ich schon wieder unpraktisch dann. (weiblich, 17 Jahre, Stadt, Adaptiv-Pragmatische)

Über Abrechnungsmodelle und Kosten ist man im Detail nicht im Bilde, vermutet aber, "dass es sich schon lohnen wird" - im Vergleich zu den Kosten des Unterhalts eines eigenen Autos oder zu Taxifahrten. In der Kostenersparnis sieht man zunächst auch den Hauptanreiz für die Nutzung von Car-Sharing-Angeboten. Unterschwellig wird aber auch deutlich, dass viele von der cleveren Nutzungsform des Teilens fasziniert sind. Umweltaspekte werden ebenfalls genannt. Die Möglichkeit, über Car-Sharing verschiedene Automodelle kennen zu lernen, wird am Rande positiv hervorgehoben. Dass die Autos aus den Car-Sharing-Pools einwandfrei gewartet und in generell gutem Zustand sind, setzen die Jugendlichen in der Regel voraus.

(1) Ich finde das gut, weil das ist deutlich billiger als ein Taxi. Und ein Kumpel von mir, wir sind auch schon mal in so einem Auto gefahren, weil die Bahn eben gestreikt hat. Und das ist auf jeden Fall eine gute Idee. Vor 
allem, weil das sind ja auch Elektroautos. Das heißt, die Umwelt wird dadurch auch noch geschont. (männlich, 16 Jahre, Stadt, Materialistische Hedonisten)

( Der Vorteil ist, dass nicht so viele Autos rumfahren. Es ist begrenzter, auch in Sachen Umwelt und Schadstoffe. Und das Auto steht selten nutzlos rum. (männlich, 15 Jahre, Land, Sozialökologische)

( Spritkosten sind weniger. Umweltverschmutzung wäre auch weniger. (männlich, 14 Jahre, Stadt, Materialistische Hedonisten)

(8) Das finde ich eigentlich richtig gut. Ich weiß jetzt nicht, wie das Preisverhältnis ist, aber es wird wohl billiger sein als wenn man sich ein eigenes Auto kauft. Das finde ich super. Wenn man nicht dauernd Autofahren muss, also wenn man nicht unbedingt ein eigenes Auto braucht, gibt es ja trotzdem Situationen, in denen man es braucht. Und wenn man den Führerschein hat, ist das Carsharing dann perfekt. Das ist eine gute Idee. (weiblich, 17 Jahre, Stadt, Adaptiv-Pragmatische)

(7) Finde ich eigentlich ganz cool. Ist bestimmt spannend, mal ein anderes Auto, das man sich vielleicht nicht leisten könnte mal zu fahren. Das ist eigentlich eine coole Idee. (männlich, 17 Jahre, Stadt, Adaptiv-Pragmatische)

(7) Wenn die Autos in gutem Zustand sind und auch immer in Ordnung gehalten werden, wäre es für mich ganz in Ordnung. Ich finde die Idee sogar sehr gut. Ich weiß natürlich nicht, wie hoch die Kosten sind. Ich glaube aber, das wäre trotzdem billiger. Man nutzt das Auto nur, wenn man es braucht. Ich finde das sehr gut. Auch wenn ich bisher noch nie etwas davon gehört habe. (männlich, 16 Jahre, Land, Adaptiv-Pragmatische)

Car-Sharing kann auch eine attraktive Übergangslösung sein (v. a. während des Studiums), bis man sich ein eigenes Auto leisten kann.

Ja gut, wenn man es sich natürlich zuerst nicht leisten kann, Studenten können sich ja so etwas nicht leisten, je nachdem, ob die Eltern das zahlen oder man selbst, dann kann man natürlich so etwas machen. 
Für eine kurze Zeit. Aber sonst würde ich es nicht machen. (männlich, 15 Jahre, Land, Materialistische Hedonisten)

( Ja, doch, also vor allem für Studenten, wo man noch kein eigenes Auto hat, finde ich es eigentlich schon ziemlich gut, ehrlich gesagt. (männlich, 17 Jahre, Stadt, Experimentalistische Hedonisten)

\section{Jugendliche aus dem ländlichen Raum sind skeptischer gegenüber Car-Sharing-Angeboten.}

Trotz der grundsätzlichen Offenheit gegenüber der Car-Sharing-Idee bestehen auch einige Vorbehalte - vor allem unter Jugendlichen aus dem ländlichen Raum. Das ist vor dem Hintergrund plausibel, dass ihnen ein eigenes Auto wichtiger ist als den Jugendlichen aus der Stadt. Weil man lange unter den Defiziten des öffentlichen Personennahverkehrs hat leiden müssen, möchte man künftig möglichst flexibel und unabhängig seine Wege zurücklegen können. Zudem haben Jugendliche auf dem Land in der Regel längere Strecken zu fahren und vermuten, dass Car-Sharing-Angebote aus Kostengründen dafür nicht geeignet sind oder Car-Sharing-Modelle aufgrund der dezentraleren Infrastruktur in ländlichen Gebieten schlichtweg nicht praktikabel wären. Auch wird deutlich, dass derartige Alternativen zum eigenen Pkw nicht zur Alltagsrealität der Eltern oder des Umfelds gehören, so dass ihnen diese allenfalls in der Theorie bekannt sind und sie sich eher nicht vorstellen können, dass und wie solche Modelle in der Realität konkret funktionieren sollen.

( Ich weiß nicht, also wenn ich älter wäre, würde ich das eher nicht machen. Mir wäre es zu viel Aufwand, wenn ich mich jedes Mal anmelden müsste und genau planen müsste. Wenn man dann halt mal Typische Zitate zur Illustration schnell noch in den Supermarkt fahren will oder so, ich glaube, es ist schon praktischer, wenn man sein eigenes Auto hat, auch wenn es vielleicht teurer ist. (weiblich, 17 Jahre, Land, Expeditive)

(7) Das ist so, man wächst ja so ein bisschen damit auf und dann, dass man so generell vielleicht einfach wei $\beta$, wenn ich jetzt herauskomme aus dem Laden, dann habe ich ein Auto, mit dem ich auch woanders 
hinfahren kann. Also ich weiß nicht, wenn ich jetzt in der Stadt wohnen würde, wäre es bestimmt nochmal anders, vielleicht weil dann auch meistens schon eins dasteht, aber hier ist ein eigenes Auto schon wichtig. (weiblich, 16 Jahre, Land, Sozialökologische)

\section{Das Prinzip der Sharing Economy stößt bei Jugendlichen auf Interesse.}

Das Prinzip des Teilens statt Besitzens finden Jugendliche aus den meisten Lebenswelten generell attraktiv, nicht zuletzt aus Kostengründen. Man ist zuversichtlich, dass sich diese Idee in der Zukunft weiter durchsetzen wird. Eigene Erfahrungen hat man bislang nur mit dem Tauschen und Teilen im Privaten gemacht: Man tauscht manchmal mit den besten Freunden Kleidung, teilt mit den Geschwistern den Computer oder mit befreundeten Familien einen Schrebergarten.

Typische

Zitate zur

|llustration
Ja, das finde ich auch eine coole Idee. Also zum Beispiel ich teile mir auch häufig mit meinen Freundinnen die Klamotten und tausche sie halt, weil es halt echt cool ist, weil dann hat man halt quasi neue Sachen. Ja, sowas finde ich schon ganz cool, klar, warum auch nicht. (weiblich, 17 Jahre, Land, Adaptiv-Pragmatische)

(1) Das finde ich eigentlich eine gute Idee, gerade weil man eine Bohrmaschine jetzt zum Beispiel nicht so oft braucht. Gerade auch als Student könnte ich mir schon vorstellen, dass das sehr praktisch ist. Sonst, es kommt halt drauf an, was man gerade braucht, wie teuer es ist, wie oft man es braucht, ob man es dann vielleicht doch regelmäßiger braucht als man ursprünglich gedacht hat. (weiblich, 17 Jahre, Land, Expeditive)

Ist eigentlich praktisch. Es gibt ja auch Leute, die das mit Wohnungen machen zum Beispiel. Also für denjenigen, der das macht, rentiert sich das bestimmt. (männlich, 17 Jahre, Stadt, Adaptiv-Pragmatische)

( Ja, finde ich eigentlich schon gut. Wir haben auch jetzt einen Garten zusammen mit einer anderen Familie (...), aber ich wollte jetzt nicht 
mein Bett mit irgend jemandem teilen, also... [LACHT] Ja, ansonsten eigentlich eine gute Idee. (weiblich, 17 Jahre, Land, Sozialökologische)

Skeptisch sind noch am ehesten Jugendliche aus den traditionellen und den unterschichtigen Lebenswelten. Hier macht man sich Sorgen, dass beim Teilen vielleicht nicht sorgfältig mit den (eigenen) Dingen umgegangen wird (Konservativ-Bürgerliche), oder man findet individuellen Besitz prinzipiell attraktiver, weil er individualistische Wünsche befriedigt und gegebenenfalls auch Status markiert (Materialistische Hedonisten, Prekäre).

( Gezwungenermaßen teilen finde ich irgendwie ein bisschen scheiße. Die Person kann ja immer noch zu mir kommen und fragen: Kann ich mal deine Bohrmaschine für dieses eine Mal ausleihen? Man erwartet, wenn man es dann nutzen will, dass es funktioniert, und dann wurde es so oft benutzt, dass es, wenn man es selber braucht, kaputt ist und man losrennen muss, das wieder in Ordnung bringen. (männlich, 17 Jahre, Stadt, Konservativ-Bürgerliche)

(1) Ich kann mir nicht vorstellen, dass das alles 100 Prozent mit rechten Dingen zugeht und das alles irgendwie sicher ist. (männlich, 17 Jahre, Stadt, Materialistische Hedonisten)

I Ich bin ja nicht so, dass ich unbedingt mit jedem Wildfremden meine Sachen teilen will. Wenn, dann mit Freunden, der Familie oder Bekannten. Aber wem das gefällt und wer gerne teilt, und wer kein Problem damit hat oder auch Angst, dass es kaputtgeht. (weiblich, 16 Jahre, Land, Materialistische Hedonisten)

(7) Wenn ich etwas kaufe, dann will ich auch, dass es mir gehört. (weiblich, 16 Jahre, Stadt, Prekäre)

Typische Zitate zur Illustration 


\subsection{Berufliche Mobilität in der Zukunft}

\section{Keine uneingeschränkte Bereitschaft zu beruflicher Mobilität.}

Mobilität in der Zukunft, vor allem im beruflichen Kontext, ist für 14- bis 17-Jährige meist noch ein wenig greifbares Thema. Zwar ist man grundsätzlich bereit, hin und wieder eine Geschäftsreise auf sich zu nehmen, die meisten Jugendlichen sind sich aber einig, dass ein Leben als Berufspendler für sie nicht in Frage kommt, weil das Familienleben unter dem Beruf nach Möglichkeit nicht leiden soll. Die Vereinbarkeit von Familie und Beruf wird also im Kontext beruflicher Mobilität mitgedacht - am stärksten von jungen Frauen aus den bürgerlichen Lebenswelten (Konservativ-Bürgerliche, Adaptiv-Pragmatische). In den bildungsfernen Lebenswelten ist man oft wenig selbstbewusst mit Blick auf berufliche Auslandserfahrungen. Man fürchtet vor allem, Sprachbarrieren nicht gewachsen zu sein.

Typische

Zitate zur |llustration

Ich glaube, für den Beruf nicht so sehr, weil ich, wie gesagt, gern eine Familie gründen möchte. Ich würde dann nicht so wahnsinnig viel unterwegs sein wollen. Ich möchte dann auch Zeit für die Familie haben. Das wäre mir wichtig im Job, also dass man genug Zeit für so etwas hat. (weiblich, 17 Jahre, Stadt, Adaptiv-Pragmatische)

( Also ich würde halt nicht so gerne weg wollen. Ich glaube, eine gute Entfernung wären so umliegende Städte, weil ich hier nicht so gerne weg wollen würde. Also in einem anderen Bundesland oder so würde ich nicht so gerne arbeiten. (weiblich, 14 Jahre, Land, Konservativ-Bürgerliche)

( Aber ich denke, das ist dann halt blöd, weil ich mich ja entscheiden müsste zwischen Beruf und Familie. Und für mich ist die Familie ein total wichtiger Punkt. Zum Beispiel musste sich die Cousine meines Freundes damals auch entscheiden zwischen ihrem Beruf Modedesignerin und ihrer ersten großen Liebe. Und sie hat sich für das Modedesign entschieden. Und ich sehe jetzt immer auf Facebook, was sie postet und wo sie schon überall war. Und dann denke ich auch, geil, so 
etwas will ich ja auch machen. Aber dafür auf die Familie zu scheißen? Das ist dann, finde ich, auch nicht das Richtige. Dann habe ich lieber einen Job, der ein bisschen ruhiger ist, und ich gehe mit meiner Familie in den Urlaub. Ich fliege dann mit denen in irgendein Land und gucke mir da etwas an, anstatt immer alleine zu sein. (weiblich, 16 Jahre, Land, Adaptiv-Pragmatische)

( Ich bin schon ein Mensch, der sich gerne an Orte bindet. Und dann könnte ich mir das eigentlich auch gar nicht vorstellen, immer so rumzufliegen zu seiner Arbeit, halt keinen festen Ort zu haben. Auch gerade, wenn man eine Familie hat, ist das schon doof. (weiblich, 17 Jahre, Land, Adaptiv-Pragmatische)

$>\quad$ Beruflich würde ich nicht viel weggehen und meine Familie allein lassen. Oder die ganze Zeit mit der Familie herumreisen, das ist nicht so toll. (männlich, 17 Jahre, Stadt, Materialistische Hedonisten)

(ICh will auf keinen Fall pendeln, immer so von einer Stadt zur anderen. Am liebsten hätte ich, dass mein Arbeitsplatz auch in meiner Stadt ist und dass man mit dem Fahrrad da hinfahren könnte. (weiblich, 16 Jahre, Land, Expeditive)

Also in Deutschland könnte ich mir vorstellen, für den Beruf unterwegs zu sein, aber nicht im Ausland. Weil ich die Sprache dann nicht richtig beherrsche. (männlich, 14 Jahre, Stadt, Prekäre)

Die Idee, beruflich für ein paar Tage oder Wochen an einen anderen Ort zu reisen, finden am ehesten Expeditive reizvoll. Sie stellen sich vor, dabei die Möglichkeit zu haben, neue Orte, fremde Kulturen und spannende Menschen kennenzulernen. Auch Experimentalistische Hedonisten finden die Vorstellung interessant, dort zu „arbeiten, wo andere Urlaub machen." Aber auch in diesen Lebenswelten kann man es sich nicht vorstellen, länger von der Familie getrennt zu leben.

( Ja, das finde ich cool. Das macht mein Vater, der hat jetzt den Job gewechselt und ist dann manchmal in Istanbul. Das fände ich schon cool, wenn ich dann manchmal in anderen Ländern bin und so etwas. (männlich, 15 Jahre, Stadt, Expeditive) 
(7) Beruflich viel unterwegs zu sein finde ich schon spannend. Aber dann ist man immer von zu Hause weg. Und wenn ich dann so alleine irgendwo bin mit meinem Beruf, das will ich nicht. (weiblich, 14 Jahre, Stadt, Expeditive)

Würde ich jetzt wirklich was mit Film machen, dann würde ich eh unterwegs sein. Das wäre ganz cool. Vor allem weil man lernt total viele Leute kennen, neue Leute, verschiedene Leute. Man lernt dadurch auch verschiedene Kulturen kennen, Länder, ganz coole Locations. Man kommt halt rum. Und das finde ich cool. Aber auf der anderen Seite, wenn man halt Familie hat, dann ist das wieder doof. Wenn man alleine ist, dann ist es cool. (männlich, 17 Jahre, Stadt, Expeditive)

( Wenn ich mir einen Job vorstellen könnte, dann vielleicht irgendwas mit Designer, vielleicht mich auch selbständig machen. Zum Beispiel Spieledesign; ein Spiel selber animieren, vielleicht sogar zum Teil selber drehen. Vielleicht würde ich mir später auch vorstellen können, wegen meinem Job nach Amerika zu gehen." (weiblich, 14 Jahre, Stadt, Experimentalistische Hedonisten)

\subsection{Reisen - heute und in Zukunft}

\section{Der Wunsch, die Welt über Reisen zu entdecken, ist stark von der Lebenswelt geprägt.}

Fragt man Jugendliche, ob sie künftig gerne viel und weit reisen möchten, fallen die Antworten in den verschiedenen Lebenswelten recht unterschiedlich aus. Auch wie man den Urlaub verbringen möchte, ist lebensweltspezifisch geprägt. In den postmodernen Lebenswelten finden sich häufig die "Traveller" und „Explorer", in den bürgerlichen die "Touristen" und "Pauschalurlauber".

Bei Expeditiven, Experimentalistischen Hedonisten und teils auch bei Adaptiv-Pragmatischen - insbesondere aber bei Sozialökologischen - ist erkennbar, dass viele Jugendliche große Lust auf das Entdecken fremder Kulturen haben und individuelles Reisen reizvoll finden. Auch längere 
Auslandsaufenthalte, die das Zeitbudget klassischer Urlaubsreisen überschreiten, findet man spannend (z. B. Au Pair, Rucksackreisen, Austauschprogramme). Unter den älteren Teenagern dieses Segments ist es nicht untypisch, bereits die ersten weiteren Reisen mit Freunden - ohne Begleitung der Eltern - konkret zu planen oder sogar schon durchgeführt zu haben.

( Ich finde halt, das ist interessant mit den Kulturen, wie die Leute so leben und die Traditionen und so. Und die Menschen kennenlernen und so. Ich finde es halt interessant. Also wir sind ja irgendwie schon verwöhnt. Zum Beispiel wenn man jetzt nach Indien oder so gehen würde, da gibt es Leute, die auch mit weniger glücklich sind. Das finde ich interessant und schön eigentlich zu beobachten. (weiblich, 17 Jahre, Land, Sozialökologische)

(7) Das ist so ein Kindestraum. Irgendwo hab ich früher mal von Machu Picchu gehört, und dann wollte ich da immer hinfahren. Und wenn ich mir jetzt etwas aussuchen könnte, würde ich, glaube ich, dahin fahren. Vielleicht da hochwandern in dieses Inkadorf und mir das anschauen. Und Südamerika finde ich eh generell total interessant. (weiblich, 16 Jahre, Land, Expeditive)

(1) Ich möchte später China auf jeden Fall mal sehen. Ich möchte mal komplett anders leben. Amerika will ich auch mal vielleicht sehen. Ägypten, also ich möchte einiges sehen. Es gibt einfach so viele Möglichkeiten, Orte, die einfach wunderschön sind. (weiblich, 14 Jahre, Land, Experimentalistische Hedonisten)

(1) Ich will halt mal sehen, wie es da ist. Also so Tokio, Kuala Lumpur, so die ganzen Städte, weil es ja eine ganz andere Welt ist. Und auch mal dieses Schmutzige und so, das kann man sich ja gar nicht vorstellen, dass da nie blauer Himmel ist. Die Erfahrung finde ich ganz cool. (männlich, 16 Jahre, Stadt, Adaptiv-Pragmatische)

In den eher bürgerlich bzw. traditionell orientierten Lebenswelten entsprechen die Traumziele nicht selten den Mainstream-Klischees (Palmen, Strand). Dabei stehen vor allem Pauschalangebote und bewährte Urlaubsdestinationen hoch im Kurs. Längere Auslandsaufenthalte üben 
per se keine Faszination aus. Nach den (typischerweise zweiwöchigen) Urlauben freut man sich auch meist wieder auf zu Hause.

Typische

Zitate zur Illustration

( In die Karibik auf einem Kreuzfahrtschiff oder sowas. Auf jeden Fall kein Flugzeug. Da habe ich Angst vor. Ja, in die Karibik. Mit schönen Palmen und dem Wasser und dem Strand. (weiblich, 17 Jahre, Land, Konservativ-Bürgerliche)

( Und wenn ich ganz viel Geld hätte, würde ich auch gern in die Karibik fahren. Ich mag so etwas: Strand und türkisfarbenes Meer. (männlich, 17 Jahre, Stadt, Konservativ-Bürgerliche)

( Wenn ich länger als zwei Wochen weg bin von Zuhause, dann möchte ich schon gerne nach Hause, weil irgendwann wird es auch langweilig dort. (männlich, 16 Jahre, Land, Materialistische Hedonisten)

Jugendliche mit Migrationshintergrund besuchen in den Ferien in der Regel die Heimatländer ihrer Eltern. Je nach lebensweltlicher Grundorientierung sind aber auch diese Jugendlichen mehr oder weniger interessiert an fernen Ländern und fremden Kulturen. Auch Pilgerdestinationen werden vereinzelt von muslimischen Jugendlichen genannt.

Typische

Zitate zur Illustration

( Die Skyline von Dubai ist wunderschön. (...) Mir gefallen so große Städte. Ich würde schon gerne die Welt erkunden, wenn ich dazu finanziell wirklich die Möglichkeit hätte. Aber wenn mich jemand fragen würde, wohin willst du, das Erste war immer Türkei. Meine Familie ist dort und man fühlt sich zu Hause. (weiblich, 16 Jahre, Land, Konservativ-Bürgerliche)

( ICh würde gerne mal nach Thailand oder auf die Philippinen, da meine Mutter da herkommt. Spanien oder so, da war ich auch noch nie. So die Sachen, die man einfach mal gesehen haben muss. (weiblich, 17 Jahre, Land, Materialistische Hedonisten) 
(1) Wegen meiner Religion will ich einmal nach Mekka, also zu diesem Stein da. Also eine Reise dahin würde ich schön finden. (männlich, 16 Jahre, Stadt, Prekäre)

\subsection{Zukunft der Mobilität}

\section{Über die Zukunft der Mobilität machen sich Jugendliche noch wenig Gedanken.}

Die Zukunftsvorstellungen von Mobilität beziehen sich bei den befragten Jugendlichen zum einen auf Entwicklungen, die bereits Realität sind oder voraussichtlich bald Realität werden (zum Beispiel der automatisierte, fahrerlose öffentliche Verkehr und das selbstfahrende Auto) und zum anderen auf - medial geprägte - Science-Fiction-Phantasien (wie zum Beispiel das Beamen von Ort zu Ort). Visionäre Vorstellungen werden dabei gern mit dem Hinweis legitimiert, dass heutige Technologien vor nicht allzu langer Zeit auch noch völlig unvorstellbar waren.

( Man hat ja viele Visionen, dass dann die Autos sogar anfangen zu schweben oder so. Das klingt für viele jetzt erstmal skurril, aber wenn man überdenkt so ... Irgendwann wird es das wirklich geben, auf jeden Fall, weil früher hat man auch nicht gedacht, dass es heutzutage so was wie Smartphones geben würde. Deswegen würde ich auch so was erwarten. Also mich würde es nicht wundern, wenn es dann wirklich einmal fliegende Autos geben würde. Mit Luftstraßen, was weiß ich was. (männlich, 16 Jahre, Land, Adaptiv-Pragmatische)

( Wahrscheinlich werden die Autos immer schneller und teurer und hochmodernisierter. Und dann kommt halt doch noch irgendwann das selbstfahrende Auto. Da legt man sich halt einfach rein und schläft bis man da ist. (weiblich, 17 Jahre, Stadt, Konservativ-Bürgerliche)

Eine fundierte Auseinandersetzung mit dem Thema Zukunft der Mobilität findet aber nicht statt. Noch am ehesten präsent ist die Entwicklung des selbstfahrenden Autos. Allerdings wissen die befragten Jugendlichen 
nicht, dass die Fachwelt (Wissenschaft, Politik, Autohersteller) heute davon ausgeht, dass selbstfahrende Autos schon in absehbarer Zeit auf unseren Straßen fahren werden. Zwar hat man davon gehört, dass „in Richtung autonomer Autos" viel geforscht wird (z.B. Google), scheint aber von der Idee nicht überzeugt zu sein.

\section{Skeptischer Blick auf die Entwicklung des selbstfahrenden Autos.}

Von autonom fahrenden Autos haben die meisten Jugendlichen schon gehört. Tendenziell überwiegen aber die skeptischen und negativen Einschätzungen. Zunächst zu den positiven Aspekten dieser Innovation aus Sicht der Befragten:

- Man vermutet einen hohen Fahrkomfort und kann sich vorstellen, dass man die Zeit im Auto entspannt (z. B. mit einem Nickerchen) verbringen oder sie produktiv für den Beruf nutzen kann.

Typische Zitate zur Illustration
Geil. Man kann dann beim Autofahren schlafen, oder es ist viel einfacher, weil wenn du so eine lange Reise machst, ich sehe das ja bei meinen Eltern, die sind danach immer richtig fertig. Und es ist praktisch auch modern. (männlich, 15 Jahre, Land, Materialistische Hedonisten)

( Ich habe schon davon gehört, dass man da angeblich nebenbei noch schlafen kann. Aber das ist eine Idee, die noch weit, weit weg ist. (männlich, 15 Jahre, Stadt, Materialistische Hedonisten)

(7) Wenn man arbeiten muss, noch irgendwelche Aufgaben erledigen muss für die Arbeit, dann kann man das halt machen. Weil ich meine, du musst ja nicht fahren. Du hast Zeit für dich, kannst schlafen und so. Das ist bestimmt bequem. (männlich, 16 Jahre, Stadt, Adaptiv-Pragmatische)

( Die Idee würde ich begrüßen. Denn dann hat man viel mehr Zeit für andere Dinge. Man kann auf dem Weg zur Arbeit vielleicht noch 
schnell etwas ausfüllen oder sich unterhalten. (männlich, 15 Jahre, Land, Adaptiv-Pragmatische)

(7) Wenn man mal keinen Bock hat zu fahren. Oder wenn man eine lange Strecke zu fahren hat, wäre das cool. Dann kannst du Pause machen und schlafen, während das Auto fährt. (männlich, 15 Jahre, Stadt, Konservativ-Bürgerliche)

- Auch kann man sich vorstellen, dass selbstfahrende Autos den Verkehr sicherer machen.

(1) Also ich finde das cool. Da kann auch nicht mehr so viel passieren, so Verkehrsunfälle. Die passieren ja bloß durch die Fehler der Menschen, also größtenteils. Dem würde damit vielleicht vorgebeugt werden, wenn das alles so funktioniert. (weiblich, 16 Jahre, Stadt, Experimentalistische Hedonisten)

(7) Es gibt vielleicht weniger Unfälle und man muss sich vielleicht nicht mehr so stark konzentrieren auf die Straße. (männlich, 14 Jahre, Stadt, Prekäre)

(1) Es ist schon cool, wenn es eine Garantie gibt, dass da keine Unfälle passieren, dann ist das schon ein großer Vorteil. (männlich, 14 Jahre, Land, Materialistische Hedonisten)

Die Argumente gegen diese Innovation werden allerdings häufig mit deutlich mehr Nachdruck formuliert:

- Man kann dieser Technologie noch nicht vertrauen: Viele Jugendliche sind skeptisch, ihr Leben in die Hand einer "Maschine" zu legen und haben erst einmal mehr Vertrauen in menschliche Fahrer. Die meisten gehen davon aus, dass die Technologie noch lange nicht sicher ist, Ausfälle oder Komplikationen daher wahrscheinlich sind. Man fürchtet, dass die Software nicht stabil laufen und zudem anfällig für Systemangriffe sein könnte.

Natürlich ist das gruselig, irgendwie, dass ein Auto ohne Fahrer fährt. Und ich glaube, ich würde mich auch nicht in so ein Teil reinsetzen. Also

Typische Zitate zur |llustration

Typische Zitate zur Illustration 
vielleicht schon, aber dann halt selber fahren. Aber ich weiß nicht, ich vertraue dem nicht so. (weiblich, 15 Jahre, Land, Experimentalistische Hedonisten)

(1) Das ist nicht so meins, also ich trau solchen Autos nicht. Da kann immer was schief gehen, also wenn man jetzt auf der Autobahn ist, 200 fährt, und das grade jetzt nicht so richtig funktioniert, dann ... (...) Selber fahren macht auch mehr Spaß. (männlich, 14 Jahre, Land, Materialistische Hedonisten)

( I ch finde das schon total witzig. Aber ich glaube, das wird total viel Chaos geben. Und ich glaube auch nicht, dass die wirklich für den Alltag gemacht sind. Denn auch wenn die Sensoren haben und so etwas, wird es trotzdem Unfälle damit geben. (weiblich, 17 Jahre, Stadt, Konservativ-Bürgerliche)

( Maschinen, die können gehackt werden. Man kann nichts 100\%ig Sicheres machen oder programmieren, das geht ja nicht. Wenn dann irgendjemand auf die schlaue Idee kommt, so etwas zu hacken, dann ist das tot alles. (männlich, 14 Jahre, Stadt, Adaptiv-Pragmatische)

I Ich finde es ein bisschen komisch, sich sozusagen wirklich nur noch auf die Technik zu verlassen. Menschen können natürlich auch viele Fehler machen, aber ich weiß nicht ... Sich nur auf die Technik zu verlassen, das finde ich schon irgendwie krass. Ich kann mir vorstellen, dass es irgendwann soweit kommt. Aber noch wäre es mir ein bisschen unheimlich, ehrlich gesagt. (weiblich, 17 Jahre, Stadt, Adaptiv-Pragmatische)

- Quer durch die Lebenswelten wird befürchtet, dass der Mensch immer weniger die Hoheit über die Technik behält und durch den technischen Fortschritt mehr und mehr entmündigt wird. Man hält es für möglich, dass der Mensch zukünftig - auch mit Blick auf andere Dimensionen der Digitalisierung des Alltags - immer "fauler", gar "dümmer" wird, weil ihm das Denken von Maschinen abgenommen wird. Das selbstfahrende Auto gilt als Paradebeispiel für ein Zuviel an Erleichterung des Alltags durch Technik. Lieber möchte man das Auto selbst bedienen und eigenständige Entscheidungen treffen - auch weil gerade darin der Spaß am Autofahren liegt. Die Erfahrung, chauffiert zu werden, machen die Jugendlichen bereits jetzt bei jeder Autofahrt. 
(1) Das ist langweilig. Ich finde das richtig langweilig. Ich fahre lieber selber. Ich möchte mich noch konzentrieren müssen. Irgendwann kommt dann irgendeine Maschine, die nimmt einem noch das Denken ab. Das ist doch dann auch nicht mehr okay. (weiblich, 17 Jahre, Land, Konservativ-Bürgerliche)

$>\quad$ Ich würde lieber selber bremsen wollen. Denn man kauft sich ja ein Auto. Und man übernimmt damit Verantwortung für sich selber und für die Leute, die über die Straße gehen, und für alle anderen, die auch im Verkehr sind. Ich verlasse mich schon manchmal gern auf die Technik, aber nicht wenn es ums Leben geht, wenn es um mein eigenes oder das Leben anderer Menschen geht. Das ist einfach zu viel Verantwortung, die man versucht, der Technik anzuvertrauen. Das Risiko würde ich nicht eingehen. (weiblich, 16 Jahre, Land, Adaptiv-Pragmatische)

( Aber ich glaube, man muss dann trotzdem gucken, dass man noch selber weiß, wie alles geht. Auch gerade den Führerschein machen ist ja so, dass man es noch mit Kupplung und allem macht und kann. Das finde ich schon wichtig, dann ist man auch selber flexibler und hat dann nicht Angst davor, mit so einem Auto zu fahren, einfach weil man weiß, man kann es. (weiblich, 16 Jahre, Land, Sozialökologische)

( Also ich würde lieber selber fahren, auch wenn das funktionieren würde. Ich würde das nicht benutzen. (männlich, 15 Jahre, Stadt, Materialistische Hedonisten)

( Ich finde es schon cool, aber ich mag das Gefühl, mein Auto selber zu fahren. Ich mag nicht, dass andere Leute meine Sachen machen. (männlich, 14 Jahre, Stadt, Adaptiv-Pragmatische)

( Ich hätte wohl total Angst, dass es dann doch nicht fährt. Und ich kann das ja dann selber gar nicht steuern, wohin das fährt. Und vielleicht hat es dann gerade eine Störung. Ich hätte da total Paranoia. (weiblich, 14 Jahre, Stadt, Expeditive)

Zusammenfassend lässt sich feststellen, dass die Mehrheit der Jugendlichen heute auf die gestiegenen Mobilitätsanforderungen (physisch und mental) eher defensiv reagiert. Mit Ausnahme der postmodernen Lebenswelten ist die Mobilitätsbereitschaft begrenzt, insbesondere 
im beruflichen Umfeld. Eine nachhaltige Auseinandersetzung mit dem Thema "Zukunft der Mobilität" findet nicht statt. Entsprechend gibt es dazu auch keine ernstzunehmenden Visionen. Innovationen wie das autonom fahrende Auto werden eher skeptisch gesehen.

Die Jugendlichen heute können als "Zweckmobilisten“ charakterisiert werden. Die multimodale Verkehrsmittelnutzung ist die Regel - idealerwerweise mit digitaler Unterstützung. Die Einstellung gegenüber den verschiedenen Mobilitätsoptionen ist nüchtern, die Verkehrsmittelwahl erfolgt pragmatisch. Auch das Auto - wiewohl immer noch unverzichtbar (v. a. im ländlichen Raum) - entfaltet lediglich noch in den unterschichtigen Lebenswelten echtes Faszinationspotenzial.

Die meisten Jugendlichen sind mit dem Mobilitätsangebot grundsätzlich zufrieden - auch mit dem öffentlichen Personenverkehr, von dem gerade in der untersuchten Altersgruppe (14 bis 17 Jahre) viele abhängig sind. Kritik (Unpünktlichkeit, mangelnde Sauberkeit, Unfreundlichkeit des Personals) wird moderat vorgetragen. Wenn die Verkehrsunternehmen die Mängel abstellen und sich um noch mehr Servicequalität bemühen, wird gerade die Kundengruppe der Jugendlichen mit Wohlwollen und Dankbarkeit reagieren.

Open Access Dieses Kapitel wird unter der Creative Commons Namensnennung - Nicht kommerziell 2.5 International Lizenz (http://creativecommons.org/licenses/by-nc/2.5/deed.de) veröffentlicht, welche für nicht kommerzielle Zwecke die Nutzung, Verbreitung und Wiedergabe in jeglichem Medium und Format erlaubt, sofern Sie den/die ursprünglichen Autor(en), den Titel des Werks und die Quelle ordnungsgemäß nennen, einen Link zur Creative Commons Lizenz beifügen und im Falle einer Abwandlung durch einen entsprechenden Hinweis deutlich erkennbar machen, dass Änderungen vorgenommen wurden.

Die in diesem Kapitel enthaltenen Bilder und sonstiges Drittmaterial unterliegen ebenfalls der genannten Creative Commons Lizenz, sofern sich aus der Abbildungslegende nichts anderes ergibt. Sofern das betreffende Material nicht unter der genannten Creative Commons Lizenz steht und die betreffende Handlung nicht nach gesetzlichen Vorschriften erlaubt ist, ist auch für die oben aufgeführten nicht-kommerziellen Weiterverwendungen des Materials die Einwilligung des jeweiligen Rechteinhabers einzuholen. 